\title{
NEW TECHNIQUE IN PHYSICAL CHEMISTRY
}

\section{By Dr. H. W. Melville, Department of Physical Chemistry, Cambridge}

$\mathrm{M}$ ODERN physical chemistry has now such wide ramifications that it is not easy to summarize compactly its recent endeavours. A number of the newer aspects of the subject were discussed from the point of view of the invention of new technique at a meeting of Section B (Chemistry) of the British Association held at Dundee on September 1. The development of such technique is a vital part of physical chemistry, and many recent discoveries are a direct consequence of these advances.

There are in chemistry innumerable problems, often concerning well-known and simple systems, which have formed bones of contention for many years. No amount of theoretical treatment will provide a solution. What is needed is a simple and conclusive experiment to settle the question once and for all. The region for inventiveness is thus of wide scope. The problems which have arisen are mainly those having to do with the mechanism of chemical reactions, and there are essentially two reasons for this state of affairs. What may be called 'classical chemistry' has established the kinds of molecule which enter into reaction and also what sorts of products emerge therefrom. But it could only surmise what happened in the intermediate stage of events. Certainly it was known that some kind of chemically reactive molecules took part in the reaction. The trouble is, however, that the fraction of molecules involved is generally so minute that no method of analytical chemistry is sensitive enough to detect their presence, apart altogether from determining their nature. Thus in order to discover how reaction occurs, essentially different methods have to be developed to perform this difficult task. These problems only arise, of course, in relatively slow reactions; very fast reactions are still outside the scope of experimental inquiry, although progress is being made in selected cases. Another problem often arises once the general question of mechanism is settled. When a polyatomic molecule containing a number of similarly disposed atoms undergoes reaction, it is also necessary to know what particular atom is actually involved. This question is now settled with great success by isotopic methods when samples of relatively pure isotopes are available.

The first part of the general problem is by no means solved at present. In dealing with such minute quantities of various molecules, any method used to measure their concentration must not, of course, alter that concentration significantly, otherwise the mechanism may be so much disturbed that the results obtained are invalid. One of the best examples of the development along these lines is the use of para-hydrogen for the measurement of hydrogen atom concentrations, in those processes where atomic hydrogen is believed to play a part, as for example in the heterogeneous and homogeneous reactions of hydrides. The method has the great merit that since conversion is brought about by exchange of atoms thus :

$$
\mathrm{H}+p \cdot \mathrm{H}_{2}=o \cdot \mathrm{H}_{2}+\mathrm{H},
$$

the hydrogen atom concentration is not disturbed. It may be mentioned that atomic concentrations as low as $10^{-7} \mathrm{~mm}$. of mercury may be so measured.

Naturally the method is a comparative one in that the reaction being investigated is really compared with that of conversion. Suitable conditions can, however, be chosen to cover a very wide range of concentrations. One example must suffice to indicate the kind of problems that can be solved. Ammonia is decomposed by light of wave-length $2000 \mathrm{~A}$. to nitrogen and hydrogen, only one molecule decomposing out of four absorbing radiation. Admixture of para-hydrogen shows that the primary dissociation is to $\mathrm{H}$ and $\mathrm{NH}_{2}$, but only about one half of the molecules decompose in this way. This alone does not explain the low quantum yield. Another technique shows that part of the inefficiency of the gross process is due to some recombination of $\mathrm{H}$ and $\mathrm{NH}_{2}$ to form $\mathrm{NH}_{3}$, for the decomposition is inhibited by atomic hydrogen produced at a controlled rate and checked by the para-conversion.

Similar methods with many ingenious modifications may be applied to all homogeneous hydride reactions in which atomic hydrogen is presumed to play a part. Although the same technique may be employed in heterogeneous reactions, the addition of para-hydrogen sometimes alters the character of the adsorbed layer on the catalyst, and therefore makes the results more difficult to interpret.

In reactions involving halogens, atomic halogen frequently is the active species concerned, whether the reaction be induced thermally or photochemically. Fortunately, in view of the comparatively low energy of dissociation, the equilibrium constants for dissociation are known accurately, and thus stationary atom concentrations are easy to compute. If, however, there is a disturbance of the stationary concentration by any competing reaction, then both rate of production and rate 
of removal of atoms must be known. It is the latter quantity that is the more difficult to measure. Two methods have been developed. One consists in actually measuring the decrease in the number of halogen molecules on illuminating the system, by an optical differential technique. The other consists in a proper examination of the expansion halogen vapour undergoes on illumination, that is, the Budde effect.

The behaviour of other reactive chemical entities such as $\mathrm{OH}, \mathrm{NH}_{2}, \mathrm{CH}_{3}, \mathrm{C}_{2} \mathrm{H}_{3}$, and so on, have been examined with similar but less refined methods, and there is here much work to be done before a complete correlation of all the data can be effected. For example, the life-time of these radicals can be determined by producing them photochemically and allowing their concentration to fall in the period of darkness succeeding illum. ination. Again, owing to the minute concentration, the chemical result in any one dark period cannot be measured. But by employing rotating sectors a large number of identical decay periods may easily be integrated to provide the information required.

The labelling of atoms in a molecule by using isotopes-radioactive and non-radioactive-has now assumed a familiar place in chemical laboratories. Deuterium is employed in all suspected cases of hydrogen atom lability and for acid-base catalysis, enolization, mutarotation and the like. Isotopic oxygen, though less plentiful, provides the key to debated mechanisms of hydrolysis and the constitution of bound oxygen atoms in molecules. Already nitrogen isotopes have indicated the rate at which nitrogen molecules are severed on synthetic ammonia catalysts. In fact, progress in this field is only limited by the rate at which isotopes may be produced, and the variety of reactions by the kinds of isotopes available.

In principle, radioactive indicators are used in the same way. They have the advantage of being easy to detect in small quantity, but often have practical limitations in their short life-times. This practical disability, however, can be overcome by using more powerful neutron generators. The question of the ionization of carbon-halogen bonds has been tackled in this way, and the biological applications of radio-phosphorus are becoming widely known.

Thus within a matter of a few years a new branch of physical chemistry has come into being. The principles of the subject are now well established, and it will not be long before every branch of chemistry will derive benefit from these advances in physico-chemical technique.

\title{
THE RELATIONSHIP BETWEEN PITHECANTHROPUS AND SINANTHROPUS
}

\author{
By Dr. G. H. R. von Koenigswald, Bandoeng, and Prof. Franz Weidenreich, \\ Peking Cenozoic Research Laboratory, Peiping Union Medical Collegge, Peking
}

$\mathrm{D}$ AVIDSON BLACK had remarked on the great similarity between the first skull of Sinanthropus to be found and the Pithecanthropus skull of Trinil, a condition which induced him to see in Pithecanthropus a Hominid form closely related to Sinanthropus (1931). The additional finds of the latter, unearthed in the interval, have confirmed Black's interpretation in every respect. But on the other hand, since the Pithecanthropus finds remained restricted to that rather incomplete specimen of Trinil, absolute evidence for his true Hominid character was lacking. In such circumstances, there was no other way open but to await the discovery of additional Pithecanthropus material before definitely solving this problem.

These discoveries materialized. Following the recovery of a rather primitive infantile skull (Homo modjokertensis) in 1936 and of a lower jaw of an adult individual, one of us (G. H. R. von K.), during 1937, discovered a skull in the undoubted Trinil deposits of Sangiran. This skull, preserved up to the basal region, conforms in every respect as to size, shape, and details to Dubois's Trinil specimen. Dubois, however, opposed the attribu. tion of this skull to Pithecanthropus. Nevertheless, the details of the interior, as well as exterior surfaces of the skull, and also the skiagrams, delineating the otherwise indistinct sutures and breakage lines, show not the slightest trace of irregularity or deformation, such as would be unavoidable if the assembly of the fragments had been artificially adapted to a particular form. To this skull of Sangiran was added another skull fragment derived from the same deposits and of the same site during the summer of 1938, briefly described in Nature of October 15, 1938, p. 715, by us. We are now in a position to report on an additional Pithecanthropus find made this year. It concerns the lower part of an upper jaw of unusually large dimensions, comprising the processus alveolares of both sides with completely preserved nasal floor and palate, the complete left 